\title{
Constrictive external nitinol meshes inhibit vein graft intimal hyperplasia in nonhuman primates
}

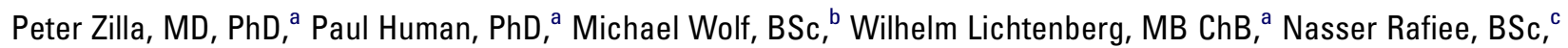 \\ Deon Bezuidenhout, $\mathrm{PhD},{ }^{\mathrm{a}}$ Nazlia Samodien, BTechHons, ${ }^{\mathrm{a}}$ Christian Schmidt, MD, ${ }^{\mathrm{a}}$ and Thomas Franz, $\mathrm{PhD}^{\mathrm{a}}$
}

From the Christiaan Barnard Department of Cardiothoracic Surgery, University of Cape Town, Cape Town, South Africa, ${ }^{a}$ the Materials and Bioscience Center, Medtronic Inc, Minneapolis, Minn, ${ }^{\mathrm{b}}$ and Medtronic Vascular, Danvers, Mass. ${ }^{c}$

The study was mainly funded through a research collaboration grant by Medtronic Inc (Minneapolis, Minn) to the University of Cape Town. The salaries of D.B., N.S., C.S., and T.F. are fully funded by the Medtronic Research Grant to the University of Cape Town, and that of P.H. is partially funded.

Re MS 07-1358, disclosure is needed, as follows: This study was supported by a grant from Medtronic; Peter Zilla and Michael Wolf hold patents on the technology described in this study; Nasser Rafie reports consulting fees from Medronic; Michael Wolf is an employee of Medtronic.

Received for publication Sept 30, 2007; revisions received Jan 23, 2008; accepted for publication Feb 15, 2008.

Address for reprints: Peter Zilla, MD, PhD, Chris Barnard Department of Cardiothoracic Surgery, University of Cape Town, 7925 Observatory, South Africa (E-mail: peter. zilla@uct.ac.za).

J Thorac Cardiovasc Surg 2008;136:717-25 $0022-5223 / \$ 34.00$

Copyright $\odot 2008$ by The American Association for Thoracic Surgery

doi:10.1016/j.jtcvs.2008.02.068
Objective: External mesh support of vein grafts has been shown to mitigate the formation of intimal hyperplasia. The aim of the present study was to address the issue of optimal mesh size in a nonhuman primate model that mimics the dimensional mismatch typically encountered between clinical vein grafts and their target arteries.

Methods: The effect of mesh size on intimal hyperplasia and endothelial preservation was assessed in bilateral femoral interposition grafts in Chacma baboons $\left(\mathrm{n}_{\Sigma}=32 / \mathrm{n}\right.$ $=8$ per mesh size). No mesh support (group I) was compared with external nitinol meshes at three different sizes: loose fitting (group II), 25\% diameter constricting (group III), and 50\% diameter constricting (group IV). Mesh sizes were seen not only in isolation but also against the background of anastomotic size mismatch at implantation, expressed as quotient of cross-sectional area of host artery to vein graft $\left(\mathrm{Q}_{\mathrm{C}}\right)$.

Results: Significant amounts of intimal hyperplasia were found in group I $\left(\mathrm{Q}_{\mathrm{C}}\right.$ median 0.20 ; intimal hyperplasia 6 weeks $=1.63 \pm 0.34 \mathrm{~mm}^{2}$; intimal hyperplasia 12 weeks $\left.=1.73 \pm 0.5 \mathrm{~mm}^{2}\right)$ and group II $\left(\mathrm{Q}_{\mathrm{C}}\right.$ median 0.25 ; intimal hyperplasia 6 weeks $=$ $1.96 \pm 1.64 \mathrm{~mm}^{2}$; intimal hyperplasia 12 weeks $=2.88 \pm 1.69 \mathrm{~mm}^{2}$ ). In contrast, group III $\left(\mathrm{Q}_{\mathrm{C}}\right.$ median 0.45 ; intimal hyperplasia 6 weeks $=0.08 \pm 0.13 \mathrm{~mm}^{2}$; intimal hyperplasia 12 weeks $\left.=0.18 \pm 0.32 \mathrm{~mm}^{2}\right)$ and IV $\left(\mathrm{Q}_{\mathrm{C}}\right.$ median 1.16 ; intimal hyperplasia 6 weeks $=0.02 \pm 0.03 \mathrm{~mm}^{2}$; intimal hyperplasia 12 weeks $=0.11 \pm 0.10$ $\left.\mathrm{mm}^{2}\right)$ showed dramatically suppressed intimal hyperplasia $(P<.01)$ at both time points. Endothelial integrity was only preserved in group IV $(P<.05)$. There were no significant differences in vascularization and inflammation in either interlayer or intergroup comparisons.

Conclusion: By using an animal model that addressed the clinical phenomenon of diameter discrepancy between vein graft and bypassed artery, we could demonstrate that suppression of intimal hyperplasia required constrictive mesh sizes.

$\mathrm{W}$ hen used as arterial bypass grafts, veins have a distinctly lower patency rate than arteries. ${ }^{1}$ The main pathologic processes behind failing vein grafts are intimal hyperplasia ${ }^{2}$ and atherosclerotic degeneration. ${ }^{3}$ Inasmuch as intimal proliferation typically precedes atherosclerotic degeneration ${ }^{4}$ it can also be seen as the "soil" for atherosclerotic vein graft changes. ${ }^{5}$ Therefore, subintimal tissue formation ultimately holds the key to the disappointing performance of vein grafts. A clinical consequence of the unsatisfactory patency rate of vein grafts was an increasing use of arterial grafts. Despite this trend, however, vein grafts remain the most widely used bypass conduits. Therefore, improving the patency of vein grafts is still a highly desirable goal. Understanding what causes the formation of vein graft intimal hyperplasia holds a key toward this goal.

Among the many factors contributing to the development of flow-limiting intimal hyperplasia, two are of central importance: low shear stress at the endothelial/blood interface $^{6}$ and high circumferential wall stress. ${ }^{7}$ In a defined system, Pouiseille's law stipulates that circumferential stress increases and shear stress decreases with 


\section{Abbreviations and Acronyms \\ ANOVA $=$ analysis of variance \\ $\mathrm{BFV}=$ baboon femoral vein \\ BSV = baboon lesser saphenous vein \\ ID = inner diameter \\ $\mathrm{OD}=$ outer diameter \\ $\mathrm{Q}_{\mathrm{C}} \quad=$ quotient of cross-sectional area of host artery to vein graft}

increasing diameter. Therefore, the relatively large diameter of clinically available vein grafts as compared with their target arteries-especially as seen in coronary surgery-is in itself a trigger for subintimal tissue proliferation. ${ }^{6,7}$ Additional dilatation of vein grafts after implantation further aggravates the situation. Vice versa, diameter reduction together with a decrease in wall tension can be expected to have an inhibitory effect on the formation of intimal hyperplasia.

As early as in 1963, Parsonnnet, Lari, and Shah ${ }^{8}$ proposed the concept of external mesh support with the goal of constricting the vein graft diameter to that of the artery, as well as preventing dilatation. Since then, the principle of vein graft protection with external meshes has been repeatedly revisited, but the multitude of animal models, materials, and diameters used resulted in opposing recommendations. ${ }^{9-11}$ To clarify a situation that has a significant potential to improve the clinical outcome of bypass surgery, we adopted a nonhuman primate model that mimicked the diameter mismatch of vein grafts and host arteries of patients. By comparing mesh sizes that created a spectrum, from loose fitting to a $50 \%$ diameter reduction, we attempted to determine an optimal size for vein grafts. Furthermore, by using the hyperelastic shape-memory material nitinol for the first time, we added a mechanical resilience factor that had not been addressed in the past.

\section{Materials and Methods Study Design}

A bilateral femoral interposition model was first established in nonhuman primates that attempted to mimic the anastomotic size mismatch of clinical vein grafts.

Subsequently, non-mesh-supported controls were compared with 3 groups of meshes: loosely size matched $(6.7 \mathrm{~mm} ; \mathrm{n}=8)$, moderately constrictive ( $\sim 25 \%$ diameter reducing; $5.0 \mathrm{~mm} ; \mathrm{n}=$ 8 ), and distinctly constrictive ( $\sim 50 \%$ diameter reducing; $3.3 \mathrm{~mm}$; $\mathrm{n}=8$ ). Grafts were analyzed after 6 and 12 weeks of arterial implantation.

The incremental approach of first pairing nonsupported control grafts with 5.0-mm mesh-supported grafts in one set of animals followed by pairing of 3.3-mm meshes with $6.7-\mathrm{mm}$ meshes in a subsequent set, followed by comparison across all 4 groups, was justified by 1-way analysis of variance (ANOVA) testing of in situ (preharvest) quotients of cross-sectional areas (Qc) of vein grafts to target arteries that confirmed a priori homogeneity of variance.

\section{Vein Source Comparison}

Chacma baboons do not have an equivalent to the human greater saphenous vein ("magna") but do have one corresponding with the lesser saphenous vein ("parva"). To determine the relevance of available baboon veins in the context of human saphenous vein grafts, histologic architecture and dimensions of human greater saphenous veins of 7 prospectively chosen patients were compared with those of the lesser saphenous veins (BSVs) and superficial femoral veins (BFVs) of 7 baboons $(30.5 \pm 4.3 \mathrm{~kg})$ unrelated to the implant group. The effect of wall compression against an external mesh was assessed by pressure fixation $(120 \mathrm{~mm} \mathrm{Hg})$ inside $3.3-\mathrm{mm}$ nitinol meshes.

\section{External Nitinol Meshes}

Nitinol wires (BB Ni-Ti alloy: nickel $56.0 \mathrm{wt} \%$, titanium 43.9365 wt $\%$, carbon $0.033 \mathrm{wt} \%$, oxygen $0.028 \mathrm{wt} \%$, hydrogen 0.0025 wt $\%$; thickness: $25 / 50 \mu \mathrm{m}$; Fort Wayne Metals, Fort Wayne, Ind) were braided using a 48-carrier braiding machine (Medtronic Vascular, Danvers, Mass [3.3 $\mathrm{mm}$ and $6.7 \mathrm{~mm} / 50 \mu \mathrm{m}$ ] and a 72 -carrier braiding machine (Prodesco Secant Medical, Perkasie, Pa [5.0 mm/ $25 \mu \mathrm{m}])$. Comparable wire density was achieved in all 3 diameters by choosing 24,36 , and 48 wires per circumference for the 3.3$\mathrm{mm}, 5.0-\mathrm{mm}$, and $6.7-\mathrm{mm}$ braid. The braid angle and pitch were $125^{\circ}$ and $0.304 \mathrm{~mm}$, respectively. Compliance was measured by circumferential tensile testing (Instron 5544 [Instron, Norwood, Mass], distilled water, $37^{\circ} \mathrm{C}$ ). All 3 mesh diameters had a negligible compliance at $120 / 80 \mathrm{~mm} \mathrm{Hg}$ of $1.14 \% \pm 0.065 \% / 100 \mathrm{~mm} \mathrm{Hg}(3.3$ $\mathrm{mm}), 2.06 \% \pm 0.04 \% / 100 \mathrm{~mm} \mathrm{Hg}(5.0 \mathrm{~mm})$, and $2.19 \% \pm$ $0.12 \% / 100 \mathrm{~mm} \mathrm{Hg}(6.7 \mathrm{~mm})$. The mesh diameters at 120/80 mm $\mathrm{Hg}$ filling pressures were calculated from the nonpressurized diameters $(0 \mathrm{~mm} \mathrm{Hg})$ and the radial compliance of each mesh size, assuming a linear increase in diameter with an increase in pressure. The inner diameters of the meshes at $120 / 80 \mathrm{~mm} \mathrm{Hg}$ were 3.39 / $3.37 \mathrm{~mm}$ (3.3-mm meshes), 5.12/5.08 $\mathrm{mm}$ (5.0-mm meshes), and $6.86 / 6.80 \mathrm{~mm}$ (6.7-mm meshes). Mesh dilatation at explantation was determined on the basis of cross-sectional image analysis of resinembedded, pressure-fixed midgraft cross sections (Nikon E 1000 $0.5 \times$ macro-lens/Leica QWin Pro V2.5; Nikon, Tokyo, Japan; Leica Microsystems, Wetzlar, Germany) pressure-fixed at $120 \mathrm{~mm} \mathrm{Hg}$.

\section{Experimental Surgery}

Adult chacma baboons $(\mathrm{n}=16 ; 18.1 \pm 0.9 \mathrm{~kg})$ were provided by the breeding and quarantine facilities of the South African Medical Research Council. All experiments were approved by the Health Sciences Faculty Animal Ethics Committee of the University of Cape Town. Surgery and preoperative and postoperative care complied with the "Principles of Laboratory Animal Care" and the "Guide for the Care and Use of Laboratory Animals" (NIH publication No. 86-23). Under general anesthesia, reversed superficial femoral veins - taken between the adductor canal and the confluence with the deep femoral vein - were used as bilateral femorofemoral interposition grafts. In the mesh-supported groups, the veins had been gently pulled through an introduction tube inside the mesh before grafting.

\section{Macroscopic Graft Dimensions}

At implantation, measurements were taken with a caliper in the middle of the segment assigned for excision or after interposition grafting using a "no-touch" technique. Arteries and grafts were 
measured during systolic vessel expansion. The quotient of crosssectional areas $\left(Q_{C}=a_{h} / a_{g}\right.$ whereby $a_{h}$ represents the cross-sectional area of the host vessel and $a_{g}$ that of the interposition graft) was chosen to express the dimensional mismatch between vein graft and host artery and indirectly as a reflection of flow velocity.

At explantation, grafts were perfusion-fixed (10\% formalin/120 $\mathrm{mm} \mathrm{Hg}$ ) before they were resected en bloc. Midgraft sections underwent macroscopic image analysis for luminal dimensions (QWinPro V2.5; Leica Microsystems Imaging Solutions).

\section{Light Microscopy}

Two series of 25 consecutive sections ( $3 \mu \mathrm{m}$; nitinol wires removed) were cut for paraffin histology. The series were approximately 300 $\mu \mathrm{m}$ apart and used for light microscopy (modified Movat's, elastic Masson's trichrome, and picrosirius red for diascopic polarization microscopy of collagen/Nikon E1000M and a Nikon Coolscope) and immunofluorescence (CD31; FVIII:rAg; Actin/Desmin; CD 68; Antibody8740/MAC387 and Ki-67 with a nuclear Dapi counterstain/Nikon 90i). Non-dewired midgraft sections were infiltrated with Technovit T8100 resin (Heraeus-Kulzer, Wehrheim, Germany) and cut with an Isomet Precision Saw (Buehler, Dusseldorf, Germany) before being stained with hematoxylin and eosin. ${ }^{12}$

\section{Electron Microscopy}

After perfusion fixation in formalin, samples for electron microscopy were postfixed in $2 \%$ glutaraldehyde in phosphate-buffered saline solution. After processing, scanning electron microscopy images were digitally captured by Orion V5.20 software (Eli, Chartleroi, Belgium) before undergoing image analysis. Transmission electron microscopy sections were viewed with a Philips TEM420 (Carl Zeiss, Veldhoven, The Netherlands) and images captured on a SIS Megaview III CCD camera (Olympus Soft Imaging Solutions GmbH, Münster Germany).

\section{Microscopic Assessment}

For image analyses, composite images were assembled from digital single frames captured at $4 \times$ or $10 \times$ magnification (Nikon Eclipse90i and Nikon Coolscope) using EclipseNet (Laboratory Imaging, Prague, Czech Republic) software. Light/polarized light toggle analyses between combined modified Masson's trichrome and picrosirius stains were used for image analysis of wall thickness and dimensions of wall layers. Intimal hyperplasia tissue was discernible both by its demarcation through a clear though sometimes fragmented internal elastic lamella and its distinctly weaker staining for actin and desmin than the adjacent media. The adventitial layer was defined between the outer demarcation of the media and the inner demarcation of the dense circumferential collagen structures characterizing the fibrous capsule. Precise mesh positions were determined on resin sections. Assessment of media muscularity, vasa vasorum, nuclear density, inflammatory cell infiltration, and cell proliferation, as well as verification of surface endothelialization, was based on immunofluorescence composite pictures. Entire wall layers were analyzed. Cellularity was related to the cross-sectional area of wall layers. Muscularity was defined as the partial cross-sectional area of smooth muscle cells within the boundaries of the media. Cellular phenomena such as cell proliferation (Ki67) and macrophage infiltration were related to the cellularity (Dapi positive nuclei per area) of each layer.
Vasa vasora (number of vessels; partial cross-sectional area) were related to cross-sectional areas of wall layers (Figure 1). For the assessment of surface endothelialization, the presence of endothelial cells on the blood surface was first immunohistochemically confirmed (CD31) before image analysis (Scion Image software; National Institutes of Health, Bethesda Md) of scanning electron micrographs was used for quantification.

\section{Statistical Analysis}

All statistical analyses were performed with the JMP6.0.2 (SAS, Inc, Cary, NC) application on an Apple PowerPC-based Macintosh computer (Apple, Cupertino, Calif). All continuous numerical variables were expressed as means \pm sample standard deviation, unless otherwise stated.

For assessment of the differences between vein types, 1-way ANOVA of layer dimensions was followed by Tukey-Kramer honestly significant difference post hoc analysis.

Analysis of change in cross-sectional luminal areas excluded preimplant values from inferential analyses of the remaining time points since these referred to unrestricted in situ veins.

Comparison of layer dimensions before and after external mesh support was performed by the Student $t$ test with 1-tailed $P$ values being reported. Nonparametric testing using the Wilcoxon rank sum test was used for comparison of $\mathrm{Q}_{\mathrm{C}}$ data between groups. ANOVA testing was used for the analysis of the 3 time points (implant, 6 weeks, and 12 weeks) with the Dunnet $t$ test being used for post hoc analysis of values at 6 and 12 weeks versus implant control values. Similarly, cross-sectional anatomic layer areas were examined by ANOVA testing with post hoc between-group comparisons using the Student $t$ test with Bonferonni correction of 2-tailed $P$ values for multiple comparisons. Square root transformation of intimal hyperplasia area data was performed following the ShapiroWilk testing for normality. Patency of grafts was examined using Fisher's exact test.

\section{Results}

\section{Validation of Vein Source}

Arterially distended BSVs were almost size matched with the superficial femoral arteries $\left(\mathrm{Q}_{\mathrm{C}}=0.89 \pm 0.31\right)$, whereas BFVs were distinctly larger $\left(\mathrm{Q}_{\mathrm{C}}=0.22 \pm 0.04\right)$. The difference in $\mathrm{Q}_{\mathrm{C}}$ was highly significant $(P<.0001$, Student $t$ test $)$. The overall wall thickness of BFVs $(576.9 \pm 174.3 \mu \mathrm{m}$ unsupported/393.9 $\pm 174.3 \mu \mathrm{m}$ mesh supported) was comparable with that of human greater saphenous veinss (562.6 \pm 231.2 unsupported/436.7 $\pm 137.4 \mu \mathrm{m}$ mesh supported) whereas that of the BSVs (317.0 $\pm 59.3 \mu \mathrm{m}$ unsupported/ $206.7 \pm 55.4 \mu \mathrm{m}$ mesh supported) was significantly less $(P=.028 / P=.009)$.

The histologic comparison of vessel wall layers showed the media of both baboon veins to be of similar composition and only mildly thinner than that of the human greater saphenous vein $(23 \% \mathrm{BSV} ; P=$ not significant; $24 \% \mathrm{BFV} ; P=$ not significant). Furthermore, muscular contraction seemed to enable smooth caliber adjustment of the veins. None of the veins inside nitinol meshes of half the maximal vein graft diameter showed signs of folding or buckling. 

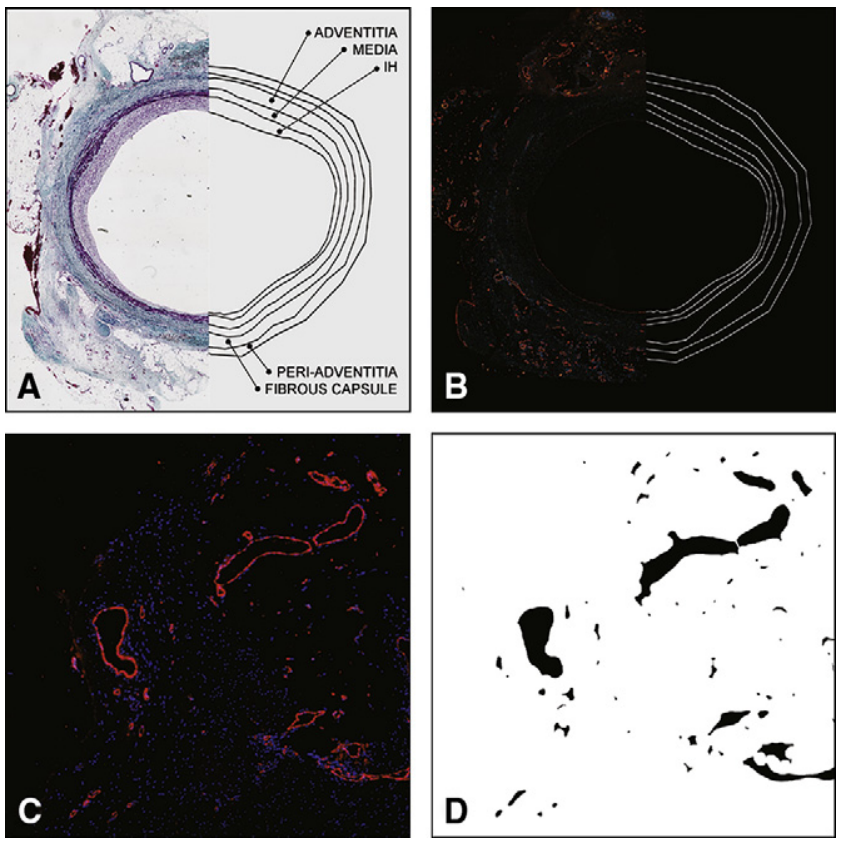

Figure 1. Image analysis of cross sections of pressure-fixed, explanted grafts by (a) manual delineation and quantification of anatomic zones on tiled stitched elastin-Masson trichrome images followed by (b) quantification of vasa vasorum within these zones on tiled factor VIII immunofluorescence images. The entire highresolution image, a section of which is shown in (c), was translated into vessel area data for each zone after hue saturation and intensity detection (d). IH, Intimal hyperplasia tissue.

Effect of External Mesh Support on Patency, Dimension, and Cross-sectional Quotient of Vein

\section{Grafts}

Graft patency was $87.5 \%$ at 6 weeks and $75.0 \%$ at 12 weeks with no significant difference between the time points and/or the groups.

On exposure to the arterial circulation, vein grafts of the unsupported control group expanded by $24 \%$ outer diameter (OD) $(P=.09)$. After a similar distention within the $6.7 \mathrm{~mm}$ meshes, the OD of vein grafts and the inner diameter (ID) of meshes were almost size matched $\left(\mathrm{OD}_{\text {Vein graft }} 3.6 \% \pm\right.$ $\left.13.3 \%<\mathrm{ID}_{\text {Mesh }}\right)$. In contrast, the $5.0-\mathrm{mm}$ and $3.3-\mathrm{mm}$ meshes led to a diameter reduction of vein grafts by $25.9 \%$ $\pm 12.3 \%$ and $48.7 \% \pm 6.6 \%$, respectively. As a result, the $\mathrm{Q}_{\mathrm{C}}$ differed significantly $(P<.0001$; Wilcoxon rank sum test) between the control group $\left(\mathrm{Q}_{\mathrm{C}}\right.$ median $=0.20$; range $0.18-0.42)$ and the $6.7-\mathrm{mm}$ meshes $\left(\mathrm{Q}_{\mathrm{C}}\right.$ median $=0.25$; range $0.23-0.47)$ on the one hand and $5.0-\mathrm{mm}\left(\mathrm{Q}_{\mathrm{C}}\right.$ median $=0.45$; range $0.43-0.47)$ and $3.3-\mathrm{mm}$ meshes $\left(\mathrm{Q}_{\mathrm{C}}\right.$ median $=1.16$; range 1.13-1.18) on the other. After 6 weeks of implantation, control veins experienced a $63 \%$ cross-sectional narrowing of the lumen $(P<.05)$ (Figures 2 and 3$)$, followed by a distinct $188 \%$ dilatation at 12 weeks $(P=.027$; Dunnet $t$

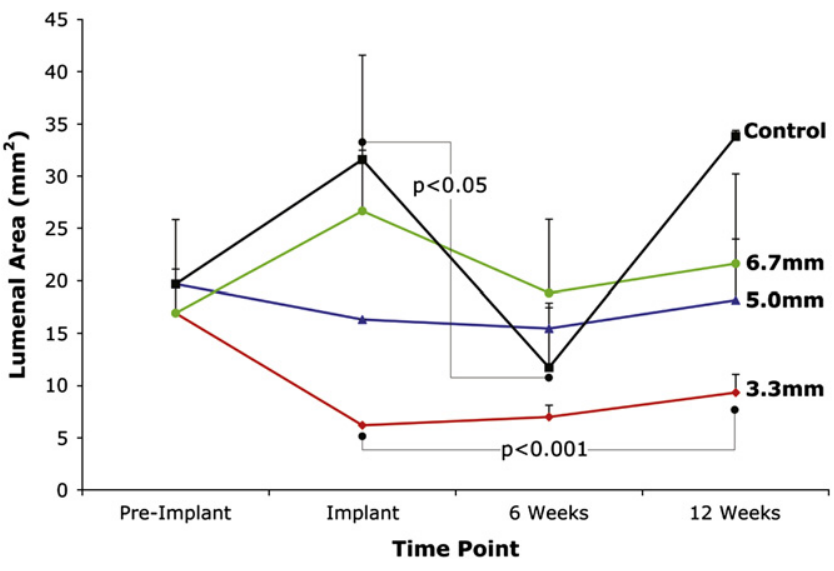

Figure 2. Change in cross-sectional luminal area. Preimplant values reflect the luminal area of in situ BSVs and were excluded from inferential statistical analysis of the remaining time points. Non-mesh-supported control grafts underwent a significant narrowing of the lumen during the initial 6 weeks followed by a marked dilatation thereafter. The mild trend toward luminal enlargement in the mesh-supported grafts is a reflection of mesh dilatation.

test) (Figures 2 and 3). Although the meshes prevented such an extreme vein graft dilatation, some degree of mesh dilatation was also observed (Figure 3).

\section{Vessel Wall Dynamics}

Macroscopic cross-sections of 6-week explants showed distinctly thickened, whitish vessel walls in both the control group and the 6.7-mm mesh-supported group (Figure 4), corresponding histologically with a dramatic increase in wall thickness $(127 \%, P<.0001$ in controls; $342 \%, P<.0001$ in 6.7-mm meshs) (Figures 5 and 6). As much as intimal proliferation was pronounced (Figure 6), it only accounted for $17.4 \% \pm 6.4 \%$ (unsupported) and $14.3 \% \pm 7.9 \%$ (6.7$\mathrm{mm}$ mesh supported) of the overall increase in wall thickness. The bulk of tissue formation took place in the fibrous capsule that "filled" the more than $0.5-\mathrm{mm}$ wide space between the mesh and the adventitia. In contrast, a relatively thin vessel wall was regularly found in the 2 mesh-supported groups that constricted the vein grafts. At 12 weeks, cross-sectional appearances remained unchanged, but histologically there was a general decrease in medial and adventitia thickness.

\section{Endothelium}

At 6 weeks, the endothelium was patchy to largely absent in controls as well as the 6.7-mm and the 5.0-mm mesh-supported groups, with no significant difference between the groups. At 12 weeks, these groups showed only a mild trend toward re-endothelialization (from $33.3 \%$ to $43.5 \%$ in controls, from $29.3 \%$ to $34.5 \%$ in $6.7-\mathrm{mm}$ meshes, and from $29.3 \%$ to $55.3 \%$ in 5.0-mm meshs; no significant difference). In contrast, vein 

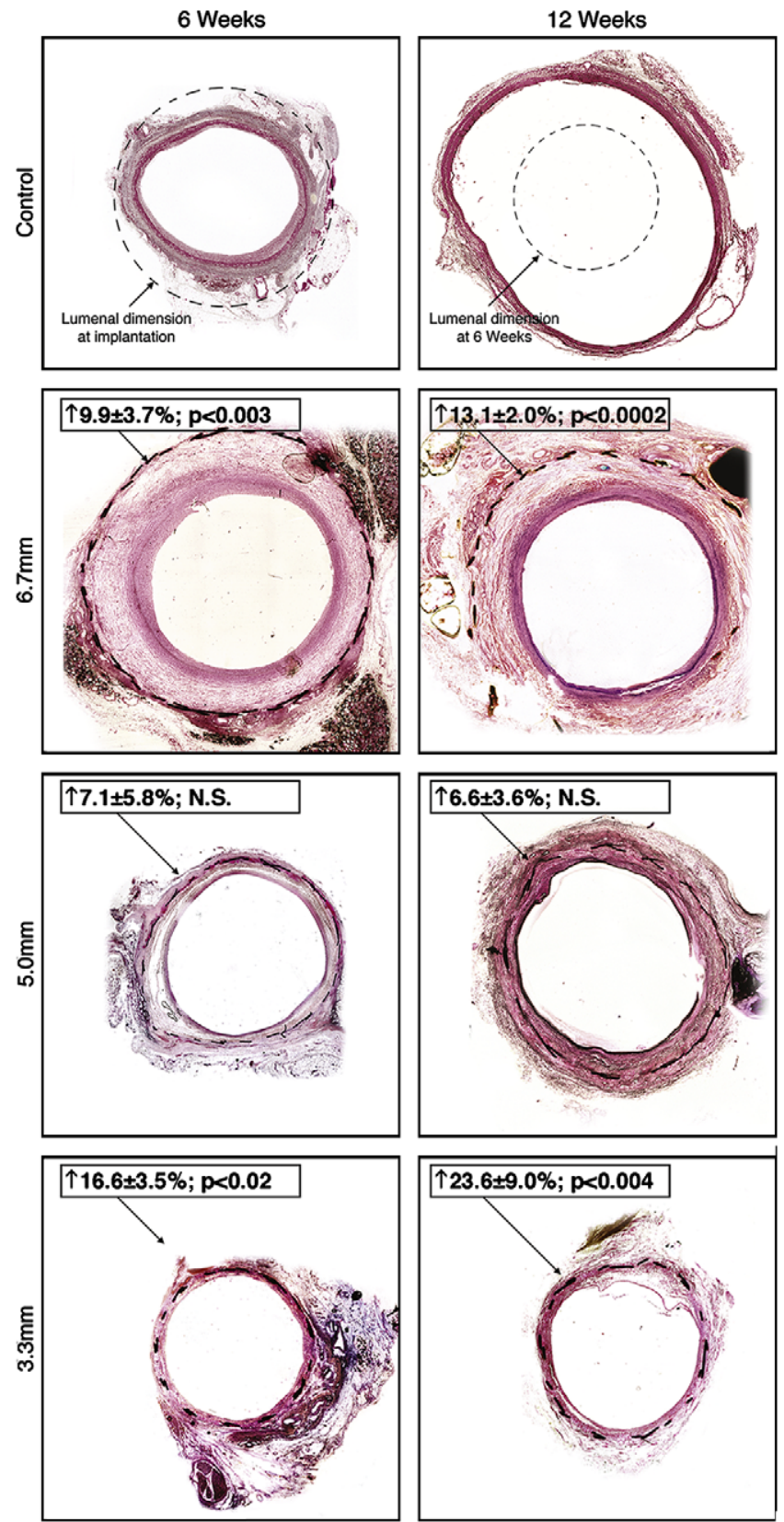

Figure 3. Non-dewired midgraft sections representing all 8 groups. (Resin; precision sawed and ground; hematoxylin and eosin stain, $0.5 \times$ lense) The circles inserted in the control group highlight the degree of shrinkage during the initial 6 weeks and the extent of dilatation thereafter. In the $6.7-\mathrm{mm}$ mesh-supported group and to a mild degree in the $5.0-\mathrm{mm}$ group, the gap between the shrunken vein grafts and the mesh is filled with fibrous tissue. Note the delicate thin wall of veins inside the $50 \%$ diameter-constrictive 3.3-mm meshes. There is a shallow longitudinal fold in the 12-week section. Numbers inserted in the left upper corner represent the percentage diameter dilatation of the meshes when compared with their dimensions at implantation. N.S., Not significant.
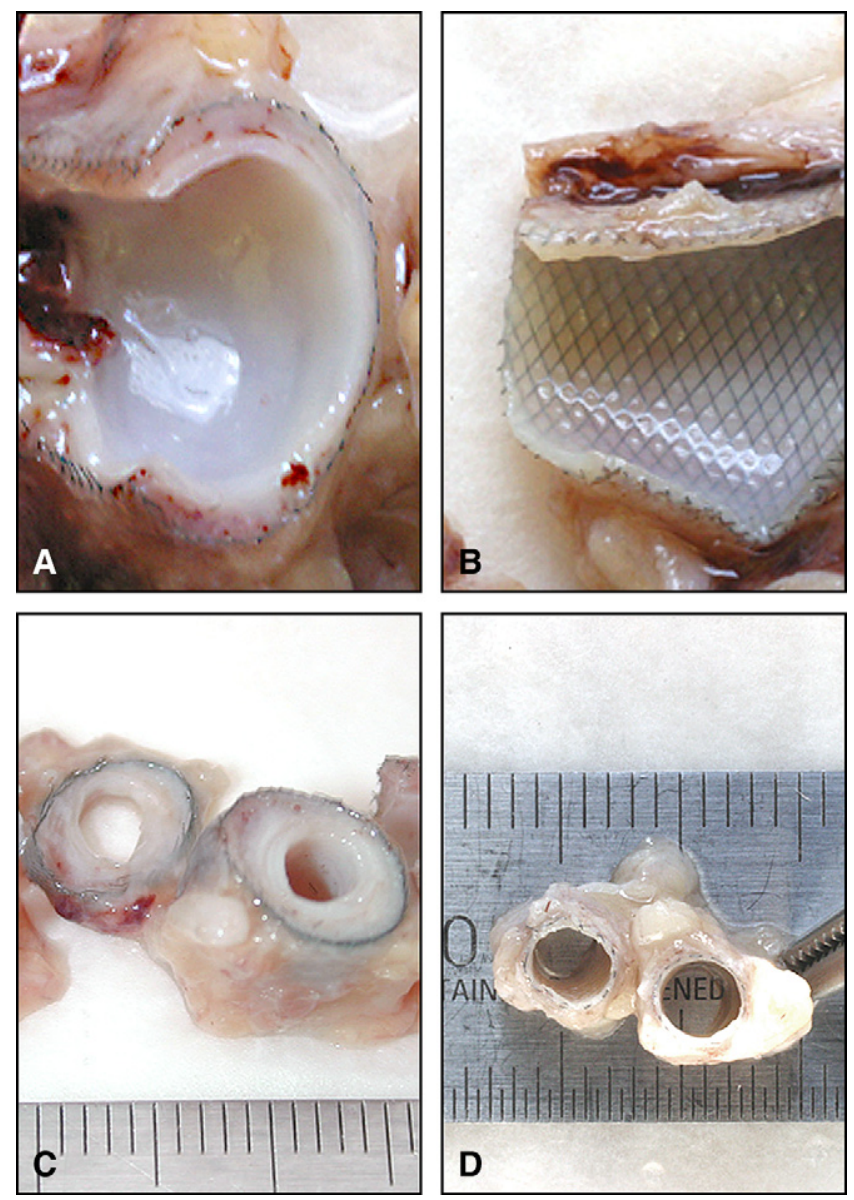

Figure 4. Macroscopic comparison of 6-week vein grafts supported by 6.7-mm meshes (left; a and c) and 3.3-mm meshes (right; b and d). In the 6.7-mm mesh-supported group, there is clear distinction between the more whitish vessel wall and the fibrous tissue filling the gap between vessel wall and mesh. Note the delicate vessel wall inside the 3.3-mm mesh.

grafts supported by 3.3-mm meshes showed a confluent endothelium differing significantly in percentage coverage (100\%) from all other groups $(P=.05)$. At 12 weeks the endothelium in the 3.3-mm mesh-supported group was still largely confluent (91.7\%; no significant difference). Ultrastructurally, the endothelium of mesh-supported grafts occasionally showed a mild ripple-mattress appearance with circumferential microfolds, a phenomenon particularly pronounced in the $3.3-\mathrm{mm}$ group. Overall, no thrombotic surface appositions were found even on endothelium-denuded surfaces.

\section{Intima}

Two typical pictures prevailed throughout: a distinct, irregular, and often half moon-shaped intimal tissue that was rich in extracellular matrix in both the control group and the $6.7 \mathrm{~mm}$ meshes as opposed to a near complete absence of 

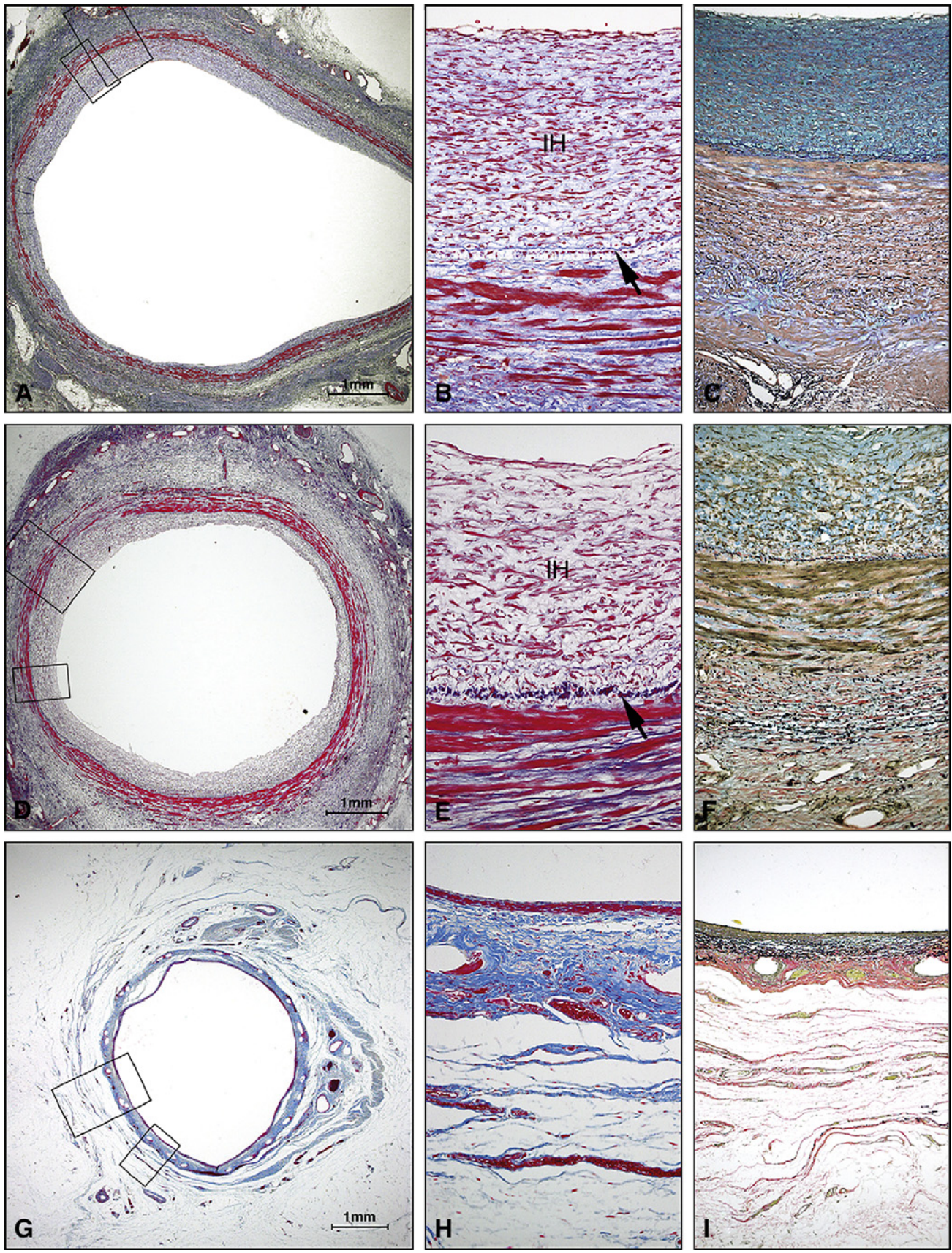

Figure 5. Serial sections of dewired midgraft regions (controls a-c/size-matched 6.7-mm mesh support: $d-f$ and $50 \%$ diameter constrictive 3.3-mm mesh support g-i) stained with modified elastic Masson's trichrome (left and middle column) and modified Movat's (right column). Small rectangles indicate high magnification areas shown in middle column $(20 \times$ objective); larger rectangles represent areas shown in right column $(10 \times$ objective). Pronounced, excentric glucosaminoglycans-rich (blue) intimal hyperplasia with discernible internal elastic lamella ( $\uparrow$ ) and loose collagen-rich media in controls and the size-matched group. Distinct $(\mathbf{5 0} \%)$ downsizing abolished intimal hyperplasia tissue (IH) and led to a thin, compacted media. 


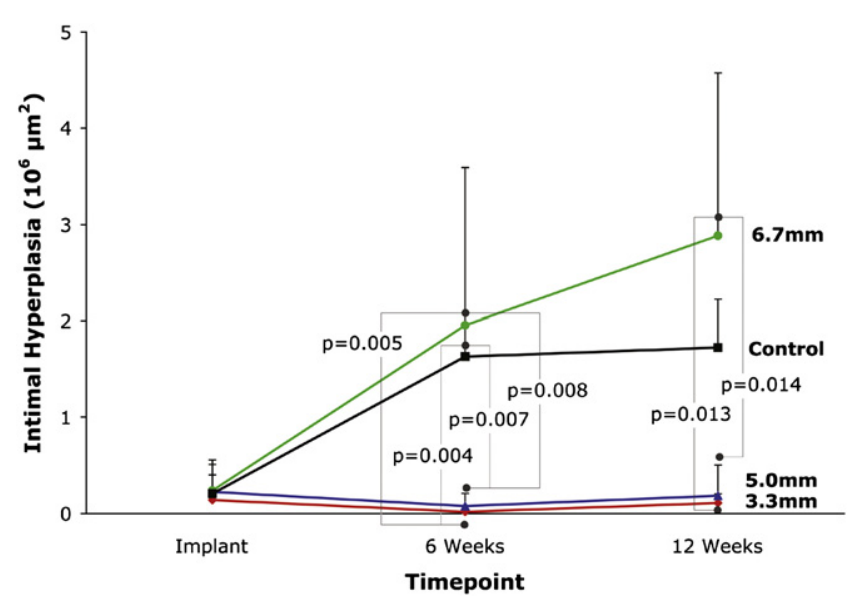

Figure 6. Cross-sectional area of intimal hyperplasia tissue. The bulk of proliferation occurs during the initial 6 weeks.

intimal tissue formation in the constrictive $3.3 \mathrm{~mm}$ and 5.0 $\mathrm{mm}$ groups. An internal elastic membrane was regularly present (Figure 5). The most dramatic increase in intimal tissue occurred in the $6.7 \mathrm{~mm}$ mesh-supported group, where it continued beyond the initial 6 weeks of implantation (Figure 6). In nonsupported controls, intimal hyperplasia appeared to reach a cross-sectional equilibrium at 6 weeks, leading to a relative thinning of the neointima at 12 weeks, corresponding with graft dilatation.

\section{Media}

The doubling of medial thickness in controls and the 6.7-mm mesh-supported group during the initial 6 weeks was associated with an actual drop in muscularity from $60.9 \% \pm 13.0 \%$ (at implant) to $33.3 \% \pm 3.3 \%$ (controls; $P=.0046$ ) and $35.9 \% \pm 3.8 \%$ (6.7-mm meshes; $P=.0226)$. Overall, a diminished total amount of smooth muscle tissue was found within the media $(-37.8 \%, P=.0015$ in controls; $-41.9 \%, P=$ not significant in 6.7-mm meshes; $-81.2 \%$, $P<.0001$ in $5.0-\mathrm{mm}$ meshes, and $-93.6 \%, P<.0001$ in 3.3-mm meshes). Intimal cells showed signs of high secretory activity on transmission electron microscopy. Remnants of platelets were found within the extracellular matrix.

\section{Adventitia, Fibrous Capsule, and Periadventitia}

The two most striking features of these outer layers were the almost absent inflammatory response against the nitinol struts (except for a few foreign body giant cells) and the surprising abundance of elastin in the mesh-supported groups (between the mesh and the media as well as relatively far outside the mesh in the periadventitial layer). The fibrous capsule was distinctly thicker in 6.7-mm meshes than in other vein grafts after 6 weeks $(280 \%$ vs controls, $P=.0052$; $198 \%$ vs $5.0-\mathrm{mm}$ meshes, $P=.0308$; and $270 \%$ vs 3.3-mm meshes, $P=.0089$ ), while no further change in capsule thickness was observed in any of the groups thereafter.

\section{Inflammatory Response}

The 6-week macrophage index of the intimal tissue was higher in the 6.7-mm group than in any other group but followed the overall trend of an abating macrophage response at 12 weeks. No significant differences were found between the groups.

\section{Cell Proliferation}

The ratio of proliferating to resting surface endothelial cells was below $1.2 \%$ in all groups except in the $6.7-\mathrm{mm}$ meshsupported group, where it was $14.4 \%$ (6 weeks) and $2.4 \%$ (12 weeks) (no significant difference). Within the wall layers, the proliferative activity did not exceed $3 \%$ at any time point. The only significantly higher proliferation index in interlayer and intergroup comparisons was found in the fibrous capsule of the 6.7-mm mesh-supported group at 6 weeks $(P=.0018)$.

\section{Vascularization}

In the 3.3-mm and 5.0-mm groups, the ingrowth of vasa vasorum was confined to layers outside the media, whereas the controls and the $6.7-\mathrm{mm}$ groups showed scanty vessels also within the media and neointima, at both 6 and 12 weeks. Inasmuch as vessel density and cross-sectional area increased over time, this trend, as well as intergroup and interlayer comparisons, were not significant.

\section{Discussion}

Our present study confirmed, in a nonhuman primate model, that the benefit of external mesh support of vein grafts is closely linked to a concomitant reduction of the size mismatch between vein grafts and host arteries.

The main underlying paradigm of the study was that the basic biomechanical principles that regulate intimal hyperplasia in general also determine the effect of external meshes. Given the fact that low shear stress and high circumferential wall stress are prime switches for the development of intimal hyperplasia ${ }^{6}$ Pouiseille's law implies that the diameter mismatch usually encountered between a large vein graft and a small host artery is a main trigger for the development of intimal hyperplasia. Inversely, it also implies that constrictive mesh sizes should counter this trigger and thus exert a suppressive effect on intimal hyperplasia.

By using the relatively small saphenous veins for interposition grafts in large arteries such as the carotids, some of the previous studies created experimental flow conditions that were distinctly different from the clinical situation. Therefore, conclusions regarding the effectiveness of loose-fitting meshes $^{11,13-15}$ are not unconditionally applicable to human bypass surgery.

In coronary artery bypass surgery, for instance, reversed saphenous veins are most often used for grafting of the 
marginal branch of the circumflex artery and the posterior descending artery.

Given the average dimension of these coronary arteries (midsection ID/cross-sectional area $2.1 \mathrm{~mm} / 3.4 \mathrm{~mm}^{2}$ and $1.8 \mathrm{~mm} / 2.5 \mathrm{~mm}^{2}$, respectively ${ }^{16}$ ), as well as that of saphenous vein grafts $(\mathrm{OD} 4.18 \pm 0.4 \mathrm{~mm}$; wall thickness 458 $\pm 17 \mu \mathrm{m}$; cross-sectional area $8.4 \mathrm{~mm}^{2} ; 200$ consecutive patients; unpublished data), the quotients of cross-sectional areas $\left(\mathrm{Q}_{\mathrm{C}}\right)$ would be in the region of 0.30 to 0.40 . Under clinical circumstances, where the runoff may be compromised, the $\mathrm{Q}_{\mathrm{C}}$ may even be below 0.30 . In contrast, if an internal thoracic artery (average OD: $3.01 \pm 0.51 \mathrm{~mm}$; wall thickness $248 \pm 108 \mu \mathrm{m}$; cross-sectional area $6.0 \pm 0.46 \mathrm{~mm}^{2}$; unpublished data) was used to graft the midsection of the left anterior descending artery (ID $2.6 \mathrm{~mm}$; cross-sectional area 5.3 $\mathrm{mm}^{2}{ }^{16}$ ), the cross-sectional quotient would be 0.88 .

In our present study, the $\mathrm{Q}_{\mathrm{C}} \mathrm{S}$ for control grafts and sizematched mesh-supported grafts were therefore similar to the $\mathrm{Q}_{\mathrm{C}} \mathrm{s}$ estimated for clinical vein grafts. Hence, it was not surprising that both the control and size-matched groups showed a degree of diffuse early intimal hyperplasia typically found in the majority of coronary bypass grafts of patients. ${ }^{17}$ In contrast, when the $\mathrm{Q}_{\mathrm{C}}$ was increased to more than 0.45 through diameter reduction of the vein graft, intimal hyperplasia was almost completely suppressed. This interdependent consideration of graft constriction and preexisting diameter mismatch between vein graft and host artery may resolve the puzzle as to why some studies reported distinctly better results with constricting meshes, ${ }^{9,10,18-21}$ while others saw a positive effect with lose-fitting meshes. ${ }^{11,13-15}$ Typically, those promoting constrictive mesh sizes used relatively large venous conduits with an estimated $\mathrm{Q}_{\mathrm{C}}$ of between 0.15 and $0.4,{ }^{9,10,18-21}$ whereas those seeing a benefit in oversizing used smaller vein grafts with an estimated $\mathrm{Q}_{\mathrm{C}}$ of up to $0.7 .^{11,13-15} \mathrm{It}$ is therefore evident that those studies advocating the oversizing of external meshes used animal models in which the vein grafts were relatively narrow when related to the runoff vessels and thus experienced higher a priori shear forces at the endothelium-blood interface. It has been shown that the threshold value for local levels of wall shear stress is 5 dynes $/ \mathrm{cm}^{2}$ below which intimal thickening occurs. ${ }^{22}$ With a $\mathrm{Q}_{\mathrm{C}}$ of as high as 0.7 , this threshold is not even remotely undercut, even if grafts remain unsupported. Therefore, constrictive mesh sizes would not have added an additional shear-stress benefit under these circumstances. The fact that differences were still seen between mesh-supported and unsupported grafts despite the high baseline $\mathrm{Q}_{\mathrm{C}}$ may be explained with a "tamponading" effect inside the mainly used Dacron grafts caused by the known seroma buildup between graft and vein. ${ }^{23}$ Thereby, even oversized meshes would turn into expansion-limiting devices. The resulting lower tangential wall stress would show an effect even if the shear forces remained subthreshold throughout.
Alternatively, different degrees of wall vascularization and/or inflammation were suggested as explanations for the protective effect of external vein support. A high vasa vasorum density was seen as a key mechanism through which loose-fitting Dacron meshes suppressed the development of intimal hyperplasia. In return, the ineffectiveness of tight-sitting Dacron meshes was explained by a lack of vasa vasora. ${ }^{15}$ The near absence of a microvessel plexus inside such meshes was ascribed to insufficient space for the development of a neoadventitia between mesh and vein graft. ${ }^{23}$ Since the delicate and wide-open nitinol meshes, which we used for external support, allowed for a well-developed vasa vasorum plexus in the neoadventitia of all groups, the previously reported effect of constrictive meshes on vasa vasorum formation may well be specific for polymeric fabrics. The fact that we observed a near-complete suppression of intimal hyperplasia suggests that vasa vasora cannot be seen as exclusive mechanisms behind the effect of external vein meshes. Similarly, the level of inflammation seen in our nitinol mesh-supported grafts was distinctly lower than that described for synthetic ${ }^{24}$ or resorbable meshes of previous studies. ${ }^{11}$ The distinct inflammatory response to Dacron was offered as one explanation for the failure of tight-sitting meshes.

However, as much as the suppression of intimal tissue proliferation is crucial in vein grafts, one must not overlook the importance of endothelial preservation. In our present study, endothelial preservation was even more affected by mesh size than intimal hyperplasia. While a $25 \%$ diameter reduction was sufficient to largely suppress intimal hyperplasia, this moderate diameter reduction led to a similar degree of early endothelial cell detachment as in control veins and the sizematched mesh-supported group. In contrast, 50\% diameter constrictions to $\mathrm{Q}_{\mathrm{C}} \mathrm{S}$ above 1.10 appeared to completely preserve the endothelium. This apparent correlation of a high $\mathrm{Q}_{\mathrm{C}}$ with endothelial preservation seems to be in accordance with most previous studies: the most pronounced difference in endothelial integrity between mesh-supported grafts and unsupported controls was found in those studies in which the baseline $\mathrm{Q}_{\mathrm{C}}$ seemed low ${ }^{9,20,25}$ and mesh support resulted in a significant constriction of the vein grafts. In contrast, when the baseline $\mathrm{Q}_{\mathrm{C}}$ was already high, no significant endothelial loss was observed whether vein grafts were mesh supported or unsupported. ${ }^{14}$ This confirms a previously suggested link between low shear stress and endothelial detachment. ${ }^{26,27}$ It could explain why, in the present study, all 3 groups with shear forces below or equal to 5 dynes $/ \mathrm{cm}^{2}$ showed substantial endothelial loss as opposed to the near complete endothelial preservation in the 50\% diameter-constricted group, where the estimated wall shear stress was higher than 20 dynes $/ \mathrm{cm}^{2}$.

Apart from intimal hyperplasia and endothelial preservation, another observation of the present study was a diminishing overall quantity of smooth muscle cells in the media. This atrophy of vascular muscle tissue puts a process often described as "arterialization" of vein grafts into perspective. Hence, 
increases in medial thickness may have been merely a reflection of a combination of bulk compression resulting from adventitial shrinkage and pronounced extracellular matrix deposition. Given the fact that veins reach their adventitial "arrest" point at as low a pressure as $30 \mathrm{~mm} \mathrm{Hg}$, loss of pulsatility may be one reason for this medial atrophy. This explanation would be supported by the even more dramatic net reduction in medial smooth muscle cells found in constrictive meshes where the noncompliant mesh absorbed the wall strain even more than the adventitial collagen in unsupported grafts.

In summary, we could demonstrate in a nonhuman primate model that moderately constrictive external vein meshes made of the hyperelastic shape-memory material nitinol are capable of almost completely abolishing the formation of intimal hyperplasia. If the goal of vein graft protection is also to preserve endothelial integrity, an even higher degree of diameter reduction seems to be required that mimics the cross-sectional area quotient between internal thoracic artery grafts and the left anterior descending coronary artery.

We thank Mrs. Melanie Black for her excellent technical assistance with resin sectioning, immuneohistochemistry, and image analysis and the Department of Anaesthesia, Groote Schuur Hospital and University of Cape Town, for anesthetic expertise for the primate procedures.

\section{References}

1. Okies JE, Page US, Bigelow JC, Krause AH, Salomon NW. The left internal mammary artery: the graft of choice. Circulation. 1984;70(3 Pt 2):I213-21.

2. Mills JL, Bandyk DF, Gahtan V, Esses GE. The origin of infrainguinal vein graft stenosis: a prospective study based on duplex surveillance. $J$ Vasc Surg. 1995;21:16-22; discussion 22-15.

3. Campeau L, Enjalbert M, Lespérance J, Vaislic C, Grondin CM, Bourassa MG. Atherosclerosis and late closure of aortocoronary saphenous vein grafts: sequential angiographic studies at 2 weeks, 1 year, 5 to 7 years, and 10 to 12 years after surgery. Circulation. 1983;68(3 Pt 2):II1-7.

4. Kohler T, Kirkman T, Gordon D, Clowes AW. Mechanism of long-term degeneration of arterialized vein grafts. Am J Surg. 1990;160:257-61.

5. Schwartz S, deBlois D, Be O. The intima. Soil for atherosclerosis and restenosis. Circ Res. 1995;77:445-65.

6. Kohler T, Kirkman T, Kraiss L, Zierler BK, Clowes AW. Increased blood flow inhibits neointimal hyperplasia in endothelialized vascular grafts. Circ Res. 1991;69:1557-65.

7. Zwolak R, Adams M, Clowes A. Kinetics of vein graft hyperplasia: association with tangential stress. J Vasc Surg. 1987;5:126-36.

8. Parsonnet V, Lari AA, Shah IH. New stent for support of veins in arterial grafts. Arch Surg. 1963;87:696-702.

9. Barra J, Volant A, Leroy J, Braesco J, Airiau J, Boschat J, et al. Constrictive perivenous mesh prosthesis for preservation of vein integrity: experimental results and application for coronary bypass grafting. J Thorac Cardiovasc Surg. 1986;92(3 Pt 1):330-6.
10. Karayannacos PE, Hostetler JR, Bond MG, Kakos GS, Williams RA, Kilman JW, et al. Late failure in vein grafts: mediating factors in subendothelial fibromuscular hyperplasia. Ann Surg. 1978;187:183-8.

11. Vijayan V, Shukla N, Johnson J, Gadsdon P, Angelini GD, Smith FC, et al. Long-term reduction of medial and intimal thickening in porcine saphenous vein grafts with a polyglactin biodegradable external sheath. J Vasc Surg. 2004;40:1011-9.

12. Rippstein P, Black M, Boivin M, Veinot JP, Ma X, Chen YX, et al. Comparison of processing and sectioning methodologies for arteries containing metallic stents. J Histochem Cytochem. 2006;54:673-81.

13. Violaris A, Newby A, Angelini G. Effects of external stenting on wall thickening in arteriovenous bypass grafts. Ann Thorac Surg. 1993;55: 667-71.

14. Izzat M, Mehta D, Bryan A, Reeves B, Newby AC, Angelini GD. Influence of external stent size on early medial and neointimal thickening in a pig model of saphenous vein bypass grafting. Circulation. 1996;94: $1741-5$.

15. Vijayan V, Smith FC, Angelini GD, Bulbulia RA, Jeremy JY. External supports and the prevention of neointima formation in vein grafts. Eur $J$ Vasc Endovasc Surg. 2002;24:13-22.

16. Dodge JT Jr, Brown BG, Bolson EL, Dodge HT. Lumen diameter of normal human coronary arteries. Influence of age, sex, anatomic variation, and left ventricular hypertrophy or dilation. Circulation. 1992;86: 232-46.

17. Lawrie GM, Lie JT, Morris GC Jr, Beazley HL. Vein graft patency and intimal proliferation after aortocoronary bypass: early and long-term angiopathologic correlations. Am J Cardiol. 1976;38:856-62.

18. Kohler T, Kirkman T, Clowes A. The effect of rigid external support on vein graft adaptation to the arterial circulation. J Vasc Surg. 1989;9: 277-85.

19. Zweep HP, Satoh S, van der Lei B, Hinrichs WL, Feijen J, Wildevuur CR. Degradation of a supporting prosthesis can optimize arterialization of autologous veins. Ann Thorac Surg. 1993;56: 1117-22.

20. Liu SQ, Moore MM, Yap C. Prevention of mechanical stretch-induced endothelial and smooth muscle cell injury in experimental vein grafts. J Biomech Eng. 2000;122:31-8.

21. Meguro T, Nakashima H, Kawada S, Tokunaga K, Ohmoto T. Effect of external stenting and systemic hypertension on intimal hyperplasia in rat vein grafts. Neurosurgery. 2000;46:963-9; discussion 969-70.

22. Sho E, Nanjo H, Sho M, Kobayashi M, Komatsu M, Kawamura K, et al. Arterial enlargement, tortuosity, and intimal thickening in response to sequential exposure to high and low wall shear stress. J Vasc Surg. 2004;39:601-12.

23. Jeremy JY, Gadsdon P, Shukla N, Vijayan V, Wyatt M, Newby AC, et al. On the biology of saphenous vein grafts fitted with external synthetic sheaths and stents. Biomaterials. 2007;28:895-908.

24. Tedoriya T, Siniawski H, Tambeur L, Huebler M, Hetzer R. Coronary artery assessment by epicardial pulsed Doppler ultrasound. Asian Cardiovasc Thorac Ann. 2001;9:257-9.

25. Zweep H, Satoh S, van der Lei B, Hinrichs WL, Dijk F, Feijen J, et al. Autologous vein supported with a biodegradable prosthesis for arterial grafting. Ann Thorac Surg. 1993;55:427-33.

26. Levesque M, Liepsch D, Moravec S, Nerem RM. Correlation of endothelial cell shape and wall shear stress in a stenosed dog aorta. Arteriosclerosis. 1986;6:220-9.

27. Repin VS, Dolgov VV, Zaikina OE, Novikov ID, Antonov AS, Nikolaeva MA, et al. Heterogeneity of endothelium in human aorta. A quantitative analysis by scanning electron microscopy. Atherosclerosis. 1984;50:35-52. 